\title{
Perspectives of Survivorship Care Plans among Older Breast Cancer Survivors: A Pilot Study
}

Jessica L. Krok-Schoen, PhD1,2, Michelle J. Naughton, PhD, MPH2,3; Anne M. Noonan, MBBChBAO2; Janell Pisegna, MOT, OTR/L; Jennifer DeSalvo, MD"; and Maryam B. Lustberg, MD²,3

${ }^{1}$ Division of Medical Dietetics and Health Sciences, School of Health and Rehabilitation Sciences, College of Medicine; ${ }^{2}$ Comprehensive Cancer Center; ${ }^{3}$ Department of Internal Medicine, College of Medicine, The Ohio State University; ${ }^{4}$ Nationwide Children's Hospital

\section{Background}

- Women aged 65 years and older account for more than half of the 3.1 million breast cancer survivors in the U.S.

- Survivorship care plans (SCPs) may improve existing and potential survivorship issues after cancer treatment.

- A focus on older breast cancer survivors is needed., as their experiences and perspectives about the value and usefulness of SCPs is limited in the literature.

Objective: To gather information about older breast cancer survivors' views of SCPs' utility and adherence to recommendations, provider communication about SCPs, and areas for SCP improvement.

\section{Methods}

Participants were eligible if they were: 65 years and older, female, diagnosed with breast cancer within the past 5 years, and received a SCP.

- This study utilized a mixed methods approach, including focus groups and self-report questionnaire data.

- The questionnaire was completed individually by participants prior to the focus group and contained questions about their health status and demographics.

- Two separate focus groups took place at the OSUCCC Stefanie Spielman Comprehensive Breast Cancer Center in February and May of 2018

- The focus groups covered participants experiences and beliefs about SCPs, the usefulness of these plans, opinions about how to improve SCPs, and barriers to following the SCP recommendations.

- Quantitative and qualitative analyses were conducted using SPSS and NVivo, respectively.

\section{Results}

- 15 out of 93 potentially eligible participants (16.1\%) took part in the two focus groups: ( $n=8$ in the first; $n=7$ in the second group).

Table 1.Participant characteristics

\begin{tabular}{|l|c|}
\hline Variables & $\%$ or mean (SD) \\
\hline Age (in years) & $62.8(10.7)$ \\
\hline Race: & $13(86.7 \%)$ \\
\hline Non-Hispanic White & $2(13.3 \%)$ \\
\hline Non-Hispanic Black & $40.2(38.6 \%)$ \\
\hline Months since diagnosis \\
\hline AJCC stage at diagnosis: \\
\hline $1 \mathrm{~A} / 1 \mathrm{~B}$ & $7(46.7 \%)$ \\
\hline $2 \mathrm{~A} / 2 \mathrm{~B}$ & $6(39.9 \%)$ \\
\hline $3 \mathrm{C}$ & $1(6.7 \%)$ \\
\hline 4 & $1(6.7 \%)$ \\
\hline
\end{tabular}

\section{Focus Group Themes}

Three major themes emerged from the focus groups:

1. Identification and Development of SCPs:

All participants received a SCP, but only 4 clearly recalled receiving the SCP and discussing it with their health care team.

"I did not realize I had one [a SCP]... and then I was going through my papers, and I did find one... so obviously it didn't make a whole lot of impression on me at the time."

\section{Communication of Survivorship Issues and Health Maintenance:}

Patients perceived poor communication with and among providers resulting in confusion and frustration about their follow-up care.

... I had an after-care thing with my surgeon and I had after-care with my oncologist, but it just seemed like they never connected on one page."

"They kind of leave out your general practitioner."

"You've had chemo and you can't think of anything, so they are telling you things and you just can't even deal with it."

\section{Suggestions to improve SCPs:}

Participants suggested education and personalization regarding the SCPs as a way to improve survivorship care. They also talked about health coaches or "navigators."

"I think it would be nice to have coaches... [who] meet you and review it."

"Someone who works with women with breast cancer"

\section{Conclusions}

Results indicated a general lack of awareness of SCPs and their importance, poor communication about SCPs, and negatively perceived coordination among providers.

- Areas identified for improvement included: clearer communication and coordination regarding survivorship care, more long-term survivorship resources, and using health coaches or navigators to facilitate adherence to SCPs.

- These findings provide insight into enhancing the content, communication, and application of SCPs to improve the survivorship experience of older breast cancer survivors. 